\title{
Clinical Study of Rhinogenic Headache and Its Management in Adults
}

\author{
Hosalli Kumaraswamy Nagarathna ${ }^{1}$, Tejaswini Honnegowda ${ }^{2}$
}

\begin{abstract}
Introduction: Rhinogenic headache is the secondary headache caused by various sinus pathologies. Aim and objective: To study various etiologies of rhinogenic headache and outcome of its management. Materials and methods: This was a prospective study on 60 patients with rhinogenic headache, presenting to the ENT department of Akash Institute of Medical Sciences and Research Centre, Karnataka, India. All patients with rhinogenic headache underwent Diagnostic Nasal Endoscopy (DNE) and X-ray of the paranasal sinuses. High-resolution computed tomography paranasal sinuses were done in patients who were to be managed surgically. Patients were managed according to the etiology by a conservative or surgical method and followed up for 6 months to evaluate the treatment outcome.

Results: All the patients were adults, predominantly males $58 \%$ and $41 \%$ were females. Fifty percent of patients were in the age group of $20-30$ years. $38.3 \%$ of patients had deviated nasal septum as the cause of rhinogenic headache, while chronic rhinosinusitis $28.3 \%$, acute rhinosinusitis $16.7 \%$, nasal polyposis 10\%, allergic rhinitis 3.3\%, and concha bullosa 3.3\%. Twenty percent of patients underwent conservative management while $80 \%$ underwent surgical management like septoplasty, inferior turbinoplasty, and functional endoscopic sinus surgery. $61.7 \%$ had complete relief from headache while $31.3 \%$ had significant relief from pain.

Conclusion: Rhinogenic headache is an important etiology of secondary headache and significantly affects the quality of life. Diagnostic difficulties do exist and the otolaryngologist plays a pivotal role in instituting appropriate treatment. The relevant treatment of the underlying etiology provides a more effective outcome in a patient with rhinogenic headache.

Keywords: Deviated nasal septum, Functional endoscopic sinus surgery, Rhinogenic headache, Rhinosinusitis, Septoplasty.

Clinical Rhinology An International Journal (2020): 10.5005/jp-journals-10013-1372
\end{abstract}

\section{INTRODUCTION}

Rhinogenic headaches are an important part of the secondary headache and in the diagnostic classification, it is categorized as headache as a result of acute, chronic, or recurrent rhinosinusitis or any other sinus pathologies expected to cause headache sinonasal pathology believed to cause a headache. ${ }^{1}$ Rhinogenic headaches are mainly concentrated in the nose and the pain related to headache and facial are primarily due to complex neurohumoral reflexes. Stammberger and Wolf classified the headache patients into three categories as follows, ${ }^{1}$ pain mediated by acute and chronic inflammation of sinus lining or barotrauma; ${ }^{2}$ headaches which are not due to sinus pathologies, but as a result of allergic and non-allergic rhinitis, trigeminal neuralgia, migraine or vascular pathologies; ${ }^{3}$ pain related to the sinonasal area, albeit the cause of inflammation is unknown. ${ }^{2}$ However, the pain in the third category are originated from the contacts and pressures between the nasal cavity adjacent mucosal surfaces when there is an existence of anatomical variations. Thus, these "contact points" between the nasal septum deformity along with middle turbinate variation, which causes pneumatization in the middle turbinate (concha bullosa) and nasal cavity lateral wall and it is the main etiological factor for the stimulation of such atypical pain. ${ }^{3}$

The rhinogenic headache is highly prevalent during acute rhinosinusitis. ${ }^{4}$ About $98 \%$ of rhinogenic headaches are mainly as a result of viral infections and sinusitis caused by bacteria accounts for $3 \% .{ }^{5}$ Various intranasal anatomic abnormalities are associated with the cause of rhinogenic headaches. These include septal deviation and spurs, concha bullosa, enlargement of the inferior
1,2 Department of ENT, Akash Institute of Medical Sciences and Research Centre, Devanahalli, Bengaluru, Karnataka, India

Corresponding Author: Tejaswini Honnegowda, Department of ENT, Akash Institute of Medical Sciences and Research Centre, Devanahalli, Bengaluru, Karnataka, India, Phone: +91 9986050155, e-mail: drtejaswini13@gmail.com

How to cite this article: Nagarathna HK, Honnegowda T. Clinical Study of Rhinogenic Headache and Its Management in Adults. Clin Rhinol An Int J 2020;13(1):5-8.

Source of support: Nil

Conflict of interest: None

turbinate, medialized middle turbinate, uncinate bulla, medially or laterally uncinate process, paradoxically middle turbinate, and large ethmoidal bulla. Among the various anatomical variations of septal deviation, septal spur, and concha bullosa, middle turbinate pneumatization is commonly observed in the middle turbinate and accounts for $50 \% .^{6}$ The etiology of headache is a multitude and it is a challenging task for an ENT specialist for its management and in the majority of the cases, rhinogenic headaches are not addressed effectively. In this backdrop, the present study was carried out to evaluate the clinical profile of rhinogenic headache and its management strategies.

\section{Materials and Methods}

This was a prospective study conducted among the 60 chronic headache patients attending the Department of Otorhinolaryngology, Akash Institute of Medical Sciences and 
Research Centre, Devanahalli, Bengaluru, Rural for a period of 2 years.

\section{Inclusion Criteria}

Male and female adult patients ( $>18$ years) and patients presenting with headache and sinonasal complaints like nasal obstruction, nasal discharge, and increased episodes of sneezing were included in the study.

\section{Exclusion Criteria}

Pediatric patients, subjects with a history of migraine, vascular headache, neuralgia, temporomandibular joint arthralgia and without any sinonasal symptoms, and geriatric patients ( $>65$ years) were excluded from the study.

The detailed history about headache included onset and duration, site of headache, nature of headache, radiation of pain, aggravating and relieving factors, treatment history, and the severity of headache based on $0-10$ on Visual Analog Scale (VAS).

The detailed history of sinonasal complaints included onset and duration, nasal obstruction, nasal discharge, sneezing episodes, postnasal discharge, decreased sense of smell, aggravating and relieving factors, and the treatment history.

Routine blood investigations were done. Patients requiring surgical management underwent high-resolution computed tomography paranasal sinuses (HRCT PNS).

Patients with a medically treatable cause like allergic rhinitis and acute rhinosinusitis underwent conservative management with antibiotics, antihistamines, and intranasal steroids.

Patients requiring surgical management underwent septoplasty, inferior tubinoplasty, and functional endoscopic sinus surgery (FESS) depending on the etiology under general anesthesia.

Patients' intensity of headache was analyzed by VAS, a psychometric response scale which was used to measure the subjective severity of headache. On a scale of $0-10$, the intensity of headache pre- and post-treatment including the follow-up was evaluated. Pretreatment score 0 suggests no pain, 1-3 mild pain, 4, 5 nagging pain, 6, 7 distressing miserable pain, 8, 9 intense dreadful horrible pain, and 10 is the worst possible unbearable excruciating pain. Post-treatment score 0 suggested that the patient was completely relieved of pain and was completely benefited from treatment. On the scale 10 suggested that there was persistent headache and not benefited and in-between the 1 and 9 on the scale that the patient was significantly improved after treatment.

\section{Statistical Analysis}

Descriptive and inferential statistics were used in this study. The continuous variables were displayed in terms of mean \pm SD and categorical variables were shown in numbers (\%). Significance was estimated at the $5 \%$ level and $p<0.05$ was considered as statistically significant. The SPSS v. 22.0 and R environment v 3.2.2. were used for the data analysis.

\section{ResUlts}

The present study was conducted on 60 patients with rhinogenic headache. Out of 60 patients, $58.3 \%$ were males and $41.7 \%$ were females. Majority of the patients were in the age group between 20 years and 30 years accounting for $50 \%$. In the age group between 31 years and 40 years, it included about $33.3 \%$ of cases. Patients within the age group between 20 and 30 displayed a male preponderance which constitutes around 57.1\%. The data were shown in Table 1.
In this study, $38.3 \%$ of patients with headaches had deviated nasal septum, $28.3 \%$ had chronic rhinosinusitis, $16.7 \%$ had acute rhinosinusitis, $10 \%$ had nasal polyposis, 3.3\% had allergic rhinitis, and $3.3 \%$ had concha bullosa. The data were shown in Table 2.

In this study, the cause of rhinogenic headache was more prevalent in the age group between 20 and 30 years (29 patients), followed by age group between 31 and 40 years (19 patients). The results were shown in Table 3.

Regarding the surgical distribution, out of 25 females, 20 patients underwent surgery and 5 patients had no surgical management $(p=0.001)$. Similarly, among the male patients out of 35 cases, 28 patients underwent surgery and 7 patients had no surgical management $(p=0.001)$. The data were shown in Table 4.

Among the 48 patients who underwent surgery, FESS was performed in the majority of the patients constituting $38.3 \%$, septoplasty was performed in $36.7 \%$, and a combination of FESS and septoplasty was done in $5 \%$.

Among the $58.3 \%$ of patients who underwent surgical management displayed complete relief from headache, while $75 \%$ of patients who underwent conservative management had complete relief of headache. $39.6 \%$ had significant relief of headache by surgical management. Only $2.1 \%$ had no benefit from surgical management. The results were shown in Table 5.

\section{Discussion}

Primary or secondary referral to otolaryngology is primarily due to pain arising from the head, face, and upper neck. The 3rd edition of the International Classification of Headache Disorders categorizes head and face pain into a primary and secondary headache. The International Headache Society (IHS) includes a category of secondary headache, which in turn includes headache attributed to acute and chronic rhinosinusitis. ${ }^{1}$ According to Third Edition of the International Classification of Headache Disorders (ICHD-3), the headache caused by acute rhinosinusitis, its severity increases when the severity of acute rhinosinusitis is also increased. Furthermore,

Table 1: Age and sex distribution of the study participants

\begin{tabular}{lccc}
\hline & \multicolumn{3}{c}{ Gender } \\
\cline { 2 - 3 } Age in years & Female & Male & Total \\
\hline$<20$ & $0(0 \%)$ & $2(5.7 \%)$ & $2(3.3 \%)$ \\
$20-30$ & $10(40 \%)$ & $20(57.1 \%)$ & $30(50 \%)$ \\
$31-40$ & $11(44 \%)$ & $9(25.7 \%)$ & $20(33.3 \%)$ \\
$41-50$ & $4(16 \%)$ & $4(11.4 \%)$ & $8(13.3 \%)$ \\
Total & $25(100 \%)$ & $35(100 \%)$ & $60(100 \%)$ \\
\hline
\end{tabular}

Table 2: Diagnosis distribution of the study participants

\begin{tabular}{lccc}
\hline & \multicolumn{3}{c}{ Gender } \\
\cline { 2 - 3 } Diagnosis & Female & Male & Total \\
\hline Allergic rhinitis & $1(4 \%)$ & $1(2.9 \%)$ & $2(3.3 \%)$ \\
$\begin{array}{l}\text { Acute rhinosi- } \\
\text { nusitis }\end{array}$ & $4(16 \%)$ & $6(17.1 \%)$ & $10(16.7 \%)$ \\
$\begin{array}{l}\text { Concha bullosa } \\
\text { Chronic rhinosi- } \\
\text { nusitis }\end{array}$ & $1(4 \%)$ & $1(2.9 \%)$ & $2(3.3 \%)$ \\
$\begin{array}{l}\text { Deviated nasal } \\
\text { septum }\end{array}$ & $9(32 \%)$ & $9(25.7 \%)$ & $17(28.3 \%)$ \\
$\begin{array}{l}\text { Nasal polyposis } \\
\text { Total }\end{array}$ & $2(8 \%)$ & $14(40 \%)$ & $23(38.3 \%)$ \\
\hline & $25(100 \%)$ & $4(11.4 \%)$ & $6(10 \%)$ \\
\hline
\end{tabular}


Table 3: Age distribution for rhinogenic headache cause

\begin{tabular}{|c|c|c|c|c|c|}
\hline Diagnosis & $<20$ years & 20-30 years & $31-40$ years & 41-50 years & Total \\
\hline Allergic rhinitis & 0 & 1 & 0 & 1 & 2 \\
\hline Acute rhinosinusitis & 0 & 8 & 2 & 0 & 10 \\
\hline Concha bullosa & 0 & 1 & 1 & 0 & 2 \\
\hline Chronic rhinosinusitis & 0 & 3 & 9 & 5 & 18 \\
\hline Deviated nasal septum & 2 & 15 & 4 & 2 & 22 \\
\hline Nasal polyposis & 0 & 1 & 3 & 2 & 6 \\
\hline Total & 2 & 29 & 19 & 10 & 60 \\
\hline
\end{tabular}

Table 4: Surgical distribution of the study participants

\begin{tabular}{lccl}
\hline & \multicolumn{2}{c}{ Gender } & \\
\cline { 2 - 3 } Surgical & Female & Male & Total \\
\hline No & $5(20 \%)$ & $7(20 \%)$ & $12(20 \%)$ \\
Yes & $20(80 \%)$ & $28(80 \%)$ & $48(80 \%)$ \\
Total & $25(100 \%)$ & $35(100 \%)$ & $60(100 \%)$ \\
$p$ value & $p=0.001$ & $p=0.001$ & \\
\hline
\end{tabular}

${ }^{*}$ Chi-square test. $p<0.05$ was considered statistically significant

Table 5: Treatment outcome in the present study

\begin{tabular}{lccl}
\hline & \multicolumn{2}{c}{ Management } & \\
\cline { 2 - 3 } Outcome & Conservative & Surgical & Total \\
\hline $\begin{array}{l}\text { Completely relieved of } \\
\text { headache }\end{array}$ & $9(75 \%)$ & $28(58.3 \%)$ & $37(61.7 \%)$ \\
No benefit & $2(16.7 \%)$ & $1(2.1 \%)$ & $3(5 \%)$ \\
Significantly improved & $1(8.3 \%)$ & $19(39.6 \%)$ & $20(33.3 \%)$ \\
Total & $12(100 \%)$ & $48(100 \%)$ & $60(100 \%)$ \\
\hline
\end{tabular}

the headaches worsen when there is a contact pressure. Meanwhile, headache associated with chronic rhinosinusitis increases or decreases along with the degree of sinus congestion and other chronic rhinosinusitis-related symptoms in paranasal sinuses. ${ }^{1}$

In this study, the headache is more prevalent in the age group between 21 years and 30 years. The majority of patients had headache in the age group of 21-30 years. Similar to our report in Kumar and Chawla, ${ }^{7}$ study $10-30$ years and Kaur and Singh, ${ }^{8}$ study 21-30 years has been reported. In Egypt, rhinogenic patients are in the age range between 18 years and 34 years. ${ }^{9}$

It is noted in the literature that the rhinogenic headache needs a multidisciplinary approach. A detailed rhinoscopic, as well as endoscopic examination, is needed for appropriate diagnosis and management. According to Altin et al., ${ }^{10}$ sinusitis, nasal polyposis, concha bullosa, and other nasal pathologies require HRCT PNS for the diagnosis and management. CT is required to rule out the pathologies that may go undetected by mere physical examination. It also helps in deciding the necessity of surgery. ${ }^{11}$ All our patients were subjected to Diagnostic Nasal Endoscopy (DNE) and 51.7\% of patients underwent X-ray PNS while $45 \%$ underwent HRCT PNS.

The deviated nasal septum was the main cause of headache followed by chronic rhinosinusitis in this study which is in correlation with Hammad and Gomaa. ${ }^{12}$ They reported septal deviation causing nasal obstruction could be the cause of the rhinogenic headache and when it is corrected by surgery, improvement in headache is observed..$^{12}$ Reports show that headache is a secondary symptom due to nasal obstruction caused by a septal deviation in rates ranging between 23 and $58 \% .{ }^{13,14}$ In line with the above studies, the main cause of headache in our study deviates nasal septum (38.3\%) and $28.3 \%$ had chronic rhinosinusitis.

Surgery is the mainstay in the management of rhinogenic headache. Huang et al. ${ }^{15}$ investigated 66 rhinogenic headache patients due to deviated nasal septum and concha bullosa and with Haller cells and surgical correction effectively decreased the frequency and intensity of headache. In our study, 3.3\% had concha bullosa and displayed significant improvement after the surgery. A contact point when present between the septum and the lateral nasal wall it causes pressure and due to which substance $P$ is released. It causes local reflexes like vasodilatation, plasma extravasation, hypersecretion, and perivascular inflammation resulting in a headache. ${ }^{10}$

Patients suffering from allergic rhinitis have turbinate hypertrophy, which causes increased mucosal contact points and an increase in severity of the headache. ${ }^{16}$ Sinonasal pathologies such as the concha bullosa, nasal polyposis, or a contact point between the nasal septum and the lateral nasal wall cause rhinogenic headache. ${ }^{10}$

Surgical correction helps in the improvement of not only nasal obstruction but also headache. ${ }^{13}$ Low and Willatt reported that out of 110 patients, $63.6 \%$ displayed complete relief of headache after the surgical correction of septal deviation. ${ }^{13}$ In a study done by Rai et al., $85 \%$ had relief of headache after undergoing septal surgery for DNS. ${ }^{17}$ In the USA patients, $76.2 \%$ had their headache scores improved by $50 \%$ or more and $42.9 \%$ were free of pain after undergoing septoplasty and endoscopic sinus surgery. ${ }^{18}$ In agreement with the above studies, $36.7 \%$ of our patients underwent septoplasty had a complete relief of headache.

In a study conducted among the Egypt headache patients, FESS displayed complete cure of $83.3 \%$ of the study participants. ${ }^{9}$ Likewise, in our study, we found $95 \%$ of patients were relieved of rhinogenic headache.

Rhinogenic headache can be managed medically or surgically depending on the etiology. Topical nasal steroids on a long-term can be used as a medical management. ${ }^{19}$ In our study, 3.3\% of patients with allergic rhinitis had not shown any significant improvement after treatment with steroid nasal spray.

Peric et al. ${ }^{19}$ and Altin et al. ${ }^{10}$ reported that a follow-up of 6 months is the optimum period to assess the rhinogenic headache treatment outcome. However, a systematic review showed that a follow-up period of $>12$ months is needed for assessing the surgical outcome in the removal of contact point as a treatment of facial pain. ${ }^{20}$ The follow-up period in our study was 6 months and we believed that it was enough time for assessing the outcome of the management of rhinogenic headache, however, longer follow-up period could also be done. 


\section{Conclusion}

Rhinogenic headaches can be treated with medical and surgical treatment. Cases of acute sinusitis, allergic rhinitis without any anatomical variations can be effectively treated by medical management. Functional endoscopic sinus surgery plays a major role in surgical management. Long-term improvement can be assessed by VAS and it also suggests that surgical management can alleviate the rhinogenic headache.

\section{Clinical Significance}

Headache and sinus diseases have many overlapping symptoms. Headache affects the quality of life and rhinogenic headache is an important etiology of secondary headache. Diagnostic difficulties do exist and awareness of clinical signs and symptoms, detailed clinical examination from the otolaryngologist plays a pivotal role in the management. Accurate surgical technique with minimal complications is very essential to alleviate the pain associated with rhinogenic headaches and also to improve the quality of life among these patients.

\section{References}

1. Headache Classification Committee of the International Headache Society (IHS). The international classification of headache disorders.3rd ed. Cephalalgia 2018;38(1):1-211. DOI: 10.1177/00034894880970s501.

2. Stammberger $\mathrm{H}$, Wolf $\mathrm{G}$. Headaches and sinus disease: the endoscopic approach. Ann Otol Rhinol Laryngol Suppl 1988;134(5_suppl):3-23. DOI: $10.1177 / 00034894880970$ s501.

3. Herzallah IR, Hamed MA, Salem SM, et al. Mucosal contact points and paranasal sinus pneumatization: does radiology predict headache causality? Laryngoscope 2015;125(9):2021-2026. DOI: 10.1002/ lary.25194.

4. De Corso E, Kar M, Cantone E, et al. Facial pain: sinus or not? Acta Otorhinolaryngol Ital 2018;38(6):485-496. DOI: 10.14639/0392-100X1721.

5. Masood A, Moumoulidis I, Panesar J. Acute rhinosinusitis in adults: an update on current management. Postgrad Med J 2007;83(980):402408. DOI: 10.1136/pgmj.2006.054767.

6. Badran HS. Role of surgery in isolated concha bullosa. Clin Med Insights Ear Nose Throat 2011;4:13-19. DOI: 10.4137/CMENT.S6769.
7. Kumar P, Chawla P. A correlative study of sinusitis versus headache. Indian J Otolaryngol Head Neck Surg 2000;52(2):125-127. DOI: 10.1007/BF03000328.

8. Kaur A, Singh A. Clinical study of headache in relation to sinusitis and its management. J Med Life 2013;6(4):389-394.

9. Kadah SS, Mokhemar S, Alkholy TE, et al. Role of endoscopy in rhinogenic contact headache not responding to medical treatment. Egypt J Otolaryngol 2019;35(3):256-261. DOI: 10.4103/ejo.ejo_6_19.

10. Altin F, Haci C, Alimoglu Y, et al. Is septoplasty effective rhinogenic headache in patients with isolated contact point between inferior turbinate and septal spur? Am J Otolaryngol 2019;40(3):364-367. DOI: 10.1016/j.amjoto.2019.02.002

11. Karataş $D$, Yüksel F, Şentürk $M$, et al. The contribution of computed tomography to nasal septoplasty. J Craniofac Surg 2013;24(5):15491551. DOI: 10.1097/SCS.0b013e3182902729.

12. Hammad MS, Gomaa MA. Role of some anatomical nasal abnormalities in rhinogenic headache. Egyptian J ENT Alli Sci 2012;13(1):31-35. DOI: 10.1016/j.ejenta.2012.01.006.

13. Low WK, Willatt DJ. Headaches associated with nasal obstruction due to deviated nasal septum. Headache 1995;35(7):404-406. DOI: 10.1111/j.1526-4610.1995.hed3507404.x.

14. Koch-Henriksen N, Gammelgaard N, Hvidegaard T, et al. Chronic headache: the role of deformity of the nasal septum. Br Med J (Clin Res Ed) 1984;288(6415):434-435. DOI: 10.1136/bmj.288.6415.434.

15. Huang HH, Lee TJ, Huang CC, et al. Non-sinusitis-related rhinogenous headache: a ten-year experience. Am J Otolaryngol 2008;29(5):326332. DOI: 10.1016/j.amjoto.2007.10.001.

16. Gryglas A. Allergic rhinitis and chronic daily headaches: is there a link? Curr Neurol Neurosci Rep 2016;16(4):33. DOI: 10.1007/s11910016-0631-z.

17. Rai U, Devi P, Singh N, et al. Contact point headache: Diagnosis and management in a tertiary care center in Northeast India. J Med Soc 2018;32(1):51-56. DOI: 10.4103/jms.jms_69_16.

18. Behin $F$, Behin B, Bigal M, et al. Surgical treatment of patients with refractory migraine headaches and intranasal contact points. Cephalalgia 2005;25(6):439-443. DOI: 10.1111/j.14682982.2004.00877.x.

19. Peric A, Rasic D, Grgurevic U. Surgical treatment of rhinogenic contact point headache: an experience from a tertiary care hospital. Int Arch Otorhinolaryngol 2016;20(2):166-171. DOI: 10.1055/s-0036-1578808.

20. Harrison L, Jones NS. Intranasal contact points as a cause of facial pain or headache: a systematic review. Clin Otolaryngol 2013;38(1):8-22. DOI: 10.1111/coa.12081. 